\title{
THE CONSERVATION OF NUMBER PRINCIPLE IN REAL ALGEBRAIC GEOMETRY
}

\author{
W. KUCHARZ \\ Department of Mathematics and Statistics University of New Mexico, Albuquerque, \\ New Mexico87131-1141 U.S.A. \\ e-mail:kucharz@math.unm.edu
}

(Received 7 December, 2001; accepted 8 November, 2002)

\begin{abstract}
The classical conservation of number principle is an important result in algebraic geometry. We present a version of this principle suitable for the study of topological properties of real algebraic varieties. Our self-contained topological proof does not depend on the intersection theory of algebraic cycles. Some applications are included.
\end{abstract}

2000 Mathematics Subject Classification. 14P25, 14C25.

1. Introduction and results. The goal of this note is to give self-contained topological proofs of certain results in real algebraic geometry, which heretofore required techniques of intersection theory (Chow rings, algebraic equivalence of cycles, etc.) $[\mathbf{1}, \mathbf{8}, \mathbf{9}]$. The main results are a suitable version of the conservation of number principle (Theorem 1.4) and an application of this principle concerning topological properties of fibers of a real algebraic morphism (Theorem 1.7).

Throughout this note the term real algebraic variety designates a locally ringed space isomorphic to an algebraic subset of $\mathbb{R}^{n}$, for some $n$, endowed with the Zariski topology and the sheaf of $\mathbb{R}$-valued regular functions. Morphisms between real algebraic varieties will be called regular maps. Basic facts on real algebraic varieties and regular maps can be found in [4]. Every real algebraic variety carries also the Euclidean topology, which is determined by the usual metric topology on $\mathbb{R}$. Unless explicitly stated otherwise, all topological notions related to real algebraic varieties will refer to the Euclidean topology.

Given a compact real algebraic variety $X$, we denote by $H_{d}^{\text {alg }}(X, \mathbb{Z} / 2)$ the subgroup of the homology group $H_{d}(X, \mathbb{Z} / 2)$ generated by the homology classes of $d$-dimensional Zariski closed subsets of $X[\mathbf{2}, \mathbf{3}, \mathbf{4}, \mathbf{6}]$. Assuming that $X$ is nonsingular, we let $H_{\text {alg }}^{c}(X, \mathbb{Z} / 2)$ denote the inverse image of $H_{d}^{\text {alg }}(X, \mathbb{Z} / 2)$ under the Poincaré duality isomorphism

$$
D_{X}: H^{c}(X, \mathbb{Z} / 2) \rightarrow H_{d}(X, \mathbb{Z}), D_{X}(\alpha)=\alpha \cap[X],
$$

where $c+d=\operatorname{dim} X$ and $[X]$ is the fundamental class of $X$.

The groups $H_{d}^{\mathrm{alg}}(-, \mathbb{Z} / 2)$ and $H_{\mathrm{alg}}^{c}(-, \mathbb{Z} / 2)$ have the expected functorial properties: If $f: X \rightarrow Y$ is a regular map between compact nonsingular real algebraic varieties, then the induced homomorphisms

$$
f_{*}: H_{*}(X, \mathbb{Z} / 2) \rightarrow H_{*}(Y, \mathbb{Z} / 2), f^{*}: H^{*}(Y, \mathbb{Z} / 2) \rightarrow H^{*}(X, \mathbb{Z} / 2)
$$


satisfy

$$
f_{*}\left(H_{d}^{\mathrm{alg}}(X, \mathbb{Z} / 2)\right) \subseteq H_{d}^{\mathrm{alg}}(Y, \mathbb{Z} / 2), f^{*}\left(H_{\mathrm{alg}}^{c}(Y, \mathbb{Z} / 2)\right) \subseteq H_{\mathrm{alg}}^{c}(X, \mathbb{Z} / 2) .
$$

Furthermore,

$$
H_{\mathrm{alg}}^{*}(X, \mathbb{Z} / 2)=\underset{c \geq 0}{\bigoplus} H_{\mathrm{alg}}^{c}(X, \mathbb{Z} / 2)
$$

is a subring of the cohomology ring $H^{*}(X, \mathbb{Z} / 2)$. Proofs of these facts are in $[\mathbf{2}, \mathbf{3}, \mathbf{6}]$ $([2,3]$ contain topological proofs).

Assume that $X$ is compact and nonsingular. A cohomology class $\alpha$ in $H_{\mathrm{alg}}^{k}(X, \mathbb{Z} / 2)$ is said to be algebraically equivalent to 0 if there exist a compact nonsingular irreducible real algebraic variety $T$, two points $t_{0}$ and $t_{1}$ in $T$, and a cohomology class $\sigma$ in $H_{\mathrm{alg}}^{k}(X \times T, \mathbb{Z} / 2)$ such that $\alpha=\sigma_{t_{1}}-\sigma_{t_{0}}$, where given $t$ in $T$, one defines $i_{t}: X \rightarrow$ $X \times T$ by $i_{t}(x)=(x, t)$ for all $x$ in $X$, and sets $\sigma_{t}=i_{t}^{*}(\sigma)$. We denote by $\operatorname{Alg}^{k}(X)$ the set of all cohomology classes in $H_{\mathrm{alg}}^{k}(X, \mathbb{Z} / 2)$ that are algebraically equivalent to 0 .

EXAMPLE 1.1. Let $X$ be a compact nonsingular irreducible real algebraic variety of dimension $n$. Obviously, $H_{\mathrm{alg}}^{n}(X, \mathbb{Z} / 2)=H^{n}(X, \mathbb{Z} / 2)$. We assert that given any two distinct points $t_{0}$ and $t_{1}$ in $X$, the cohomology class $\alpha$ in $H_{\text {alg }}^{n}(X, \mathbb{Z} / 2)$, Poincare dual to the homology class in $H_{0}^{\text {alg }}(X, \mathbb{Z} / 2)$ represented by $\left\{t_{0}, t_{1}\right\}$, belongs to $\operatorname{Alg}^{n}(X)$. Indeed, let $\sigma$ in $H_{\text {alg }}^{n}(X \times X, \mathbb{Z} / 2)$ be the cohomology class Poincare dual to the homology class in $H_{n}^{\text {alg }}(X \times X, \mathbb{Z} / 2)$ represented by the diagonal

$$
\Delta=\{(x, t) \in X \times X \mid x=t\} .
$$

For any point $t$ in $X$, the map $i_{t}: X \rightarrow X \times X$, defined by $i_{t}(x)=(x, t)$ for all $x$ in $X$, is transverse to $\Delta$ and hence $D_{X}\left(i_{t}^{*}(\sigma)\right)$ is the homology class in $H_{0}(X, \mathbb{Z} / 2)$ represented by $i_{t}^{-1}(\Delta)$. Since $i_{t}^{*}(\sigma)=\sigma_{t}$ and $i_{t}^{-1}(\Delta)=\{t\}$, we get $\alpha=\sigma_{t_{1}}-\sigma_{t_{0}}$. Thus $\alpha$ belongs to $\operatorname{Alg}^{n}(X)$ as asserted. Note that $\alpha \neq 0$ if $t_{0}$ and $t_{1}$ belong to distinct connected components of $X$.

In a straightforward manner one can prove the following result.

Proposition 1.2. For any compact nonsingular real algebraic variety $X$, the set $\operatorname{Alg}^{k}(X)$ is a subgroup of $H_{\mathrm{alg}}^{k}(X, \mathbb{Z} / 2)$. If $\alpha$ is in $\operatorname{Alg}^{k}(X)$ and $\gamma$ is in $H_{\mathrm{alg}}^{\ell}(X, \mathbb{Z} / 2)$, then $\alpha \cup \gamma$ is in $\operatorname{Alg}^{k+\ell}(X)$. If moreover, $\delta$ is in $\operatorname{Alg}^{m}(Y)$, where $Y$ is a compact nonsingular real algebraic variety, then $\gamma \times \delta$ is in $\operatorname{Alg}^{\ell+m}(X \times Y)$.

The group $\operatorname{Alg}^{k}(-)$ also has nice functorial properties.

Proposition 1.3. Let $f: X \rightarrow Y$ be a regular map between compact nonsingular real algebraic varieties. Then

(i) $f^{*}\left(\operatorname{Alg}^{k}(Y)\right) \subseteq \operatorname{Alg}^{k}(X)$,

(ii) $\left(D_{Y}^{-1} \circ f_{*} \circ D_{X}\right)\left(\operatorname{Alg}^{n-k}(X)\right) \subseteq \operatorname{Alg}^{p-k}(Y)$, where $n=\operatorname{dim} X$ and $p=\operatorname{dim} Y$.

Propositions 1.2 and 1.3 will be proved in Section 2.

Given a compact nonsingular real algebraic variety $X$, two cohomology classes $\alpha_{1}$ and $\alpha_{2}$ in $H_{\mathrm{alg}}^{k}(X, \mathbb{Z} / 2)$ are said to be algebraically equivalent if $\alpha_{1}-\alpha_{2}$ is in $\operatorname{Alg}^{k}(X)$.

For $\alpha$ in $H^{k}(X, \mathbb{Z} / 2)$ and $\beta$ in $H^{\ell}(X, \mathbb{Z} / 2)$, where $k+\ell=\operatorname{dim} X$, we denote by $\alpha \bullet \beta$ the intersection number of $\alpha$ and $\beta$, that is, $\alpha \bullet \beta:=\langle\alpha \cup \beta,[X]\rangle$. Thus $\alpha \bullet \beta$ is an element of $\mathbb{Z} / 2$. 
The next result is called the conservation of number principle.

THEOREM 1.4. Let $X$ be a compact nonsingular real algebraic variety. Assume that $\alpha_{1}, \alpha_{2}$ in $H_{\mathrm{alg}}^{k}(X, \mathbb{Z} / 2)$ are algebraically equivalent and $\beta_{1}, \beta_{2}$ in $H_{\mathrm{alg}}^{\ell}(X, \mathbb{Z} / 2)$ are algebraically equivalent. If $k+\ell=\operatorname{dim} X$, then $\alpha_{1} \bullet \beta_{1}=\alpha_{2} \bullet \beta_{2}$.

As a consequence we immediately obtain the following fact.

COROLlARY 1.5. For any compact nonsingular real algebraic variety $X$, one has

$$
\operatorname{dim}_{\mathbb{Z} / 2}\left(H^{k}(X, \mathbb{Z} / 2) / H_{\mathrm{alg}}^{k}(X, \mathbb{Z} / 2)\right) \geq \operatorname{dim}_{\mathbb{Z} / 2} \operatorname{Alg}^{\ell}(X),
$$

where $k+\ell=\operatorname{dim} X$.

Proof. By Theorem 1.4, $\alpha \bullet \beta=0$ for all $\alpha$ in $H_{\mathrm{alg}}^{k}(X, \mathbb{Z} / 2)$ and all $\beta$ in $\operatorname{Alg}^{\ell}(X)$. The proof is complete since

$$
H^{k}(X, \mathbb{Z} / 2) \times H^{\ell}(X, \mathbb{Z} / 2) \rightarrow \mathbb{Z} / 2, \quad(\alpha, \beta) \rightarrow \alpha \bullet \beta
$$

is a dual pairing [7, Proposition 8.13].

EXAMPLE 1.6. Note that

$$
X=\left\{(x, y, z) \in \mathbb{R}^{3} \mid\left(\left(x^{2}+y^{2}\right)-1\right)\left(\left(x^{2}+y^{2}\right)-2\right)+z^{2}=0\right\}
$$

is a nonsingular Zariski closed surface in $\mathbb{R}^{3}$, homeomorphic to a torus, and

$$
C=\left\{(u, v) \in \mathbb{R}^{2} \mid\left(u^{2}-1\right)\left(u^{2}-2\right)+v^{2}=0\right\}
$$

is a compact nonsingular Zariski closed curve in $\mathbb{R}^{2}$, with two connected components $C_{+}$containing $(1,0)$ and $C_{-}$containing $(-1,0)$. The map $\pi: X \rightarrow C, \pi(x, y, z)=$ $\left(x^{2}+y^{2}, z\right)$, is regular, $\pi(X)=C_{+}$, and $\pi: X \rightarrow C_{+}$is a smooth (of class $\mathcal{C}^{\infty}$ ) circle bundle over $C_{+}$. Let $\beta$ be the cohomology class in $H^{1}(C, \mathbb{Z} / 2)$ Poincaré dual to the homology class in $H_{0}(C, \mathbb{Z} / 2)$ represented by $\{(1,0),(-1,0)\}$. In view of Example 1.1, $\beta$ is in $\operatorname{Alg}^{1}(C)$. It follows from Proposition 1.3(i) that $\pi^{*}(\beta)$ belongs to $\operatorname{Alg}^{1}(X)$. By construction, $\pi^{*}(\beta) \neq 0$ and hence $\operatorname{Alg}^{1}(X) \neq 0$. Applying Corollary 1.5 , we get $H_{\mathrm{alg}}^{1}(X, \mathbb{Z} / 2) \neq H^{1}(X, \mathbb{Z} / 2)$. Since $H^{1}(X, \mathbb{Z} / 2) \cong(\mathbb{Z} / 2)^{2}$, we have $H_{\text {alg }}^{1}(X, \mathbb{Z} / 2)=\operatorname{Alg}^{1}(X) \cong \mathbb{Z} / 2$.

If $X^{n}=X \times \cdots \times X$ is the $n$-fold product, then, in view of the last statement of Proposition 1.2, $\operatorname{Alg}^{k}\left(X^{n}\right) \neq 0$ for $1 \leq k \leq n$.

This example was first used by Joost van Hamel (unpublished) to illustrate a somewhat different phenomenon.

Our next result can also be deduced from Theorem 1.4.

THEOREM 1.7. Let $f: X \rightarrow Y$ be a regular map between compact nonsingular real algebraic varieties. If $Y$ is irreducible, then given two regular values $y_{1}$ and $y_{2}$ of $f$, the smooth manifolds $f^{-1}\left(y_{1}\right)$ and $f^{-1}\left(y_{2}\right)$ are cobordant.

This result is of interest if $y_{1}$ and $y_{2}$ belong to distinct connected components of $Y$. A different proof of Theorem 1.7 can be found in [5].

Proofs of Theorems 1.4 and 1.7 are given in Section 3. 
2. Proof of the propositions. Given real algebraic varieties $X$ and $T$, a point $t$ in $T$, and a cohomology class $\tau$ in $H^{k}(X \times T, \mathbb{Z} / 2)$, we set $\tau_{t}=i_{t}^{*}(\tau)$, where $i_{t}: X \rightarrow X \times T$ is defined by $i_{t}(x)=(x, t)$ for all $x$ in $X$.

It is convenient to give the following characterization of cohomology classes algebraically equivalent to 0 .

LEMMA 2.1. For any compact nonsingular real algebraic variety $X$, given $a$ cohomology class $\alpha$ in $H_{\mathrm{alg}}^{k}(X, \mathbb{Z} / 2)$, the following conditions are equivalent:

(a) $\alpha$ is algebraically equivalent to 0 ,

(b) there exist a compact nonsingular irreducible real algebraic variety $T$, two points $t_{0}$ and $t_{1}$ in $T$, and a cohomology class $\tau$ in $H_{\mathrm{alg}}^{k}(X \times T, \mathbb{Z} / 2)$ such that $\tau_{t_{0}}=0$ and $\tau_{t_{1}}=\alpha$.

Proof. Suppose that (a) holds. Then there exist a compact nonsingular irreducible real algebraic variety $T$, two points $t_{0}$ and $t_{1}$ in $T$, and a cohomology class $\sigma$ in $H_{\text {alg }}^{k}(X \times$ $T, \mathbb{Z} / 2$ ) such that $\alpha=\sigma_{t_{1}}-\sigma_{t_{0}}$. Let $\pi: X \times T \rightarrow X$ be the canonical projection. Since $i_{t_{0}} \circ \pi \circ i_{t}=i_{t_{0}}$ for every point $t$ in $T$, setting $\tau=\sigma-\pi^{*}\left(i_{t_{0}}^{*}(\sigma)\right)$, we get

$$
\tau_{t}=i_{t}^{*}(\sigma)-i_{t}^{*}\left(\pi^{*}\left(i_{t_{0}}^{*}(\sigma)\right)\right)=\sigma_{t}-\left(i_{t_{0}} \circ \pi \circ i_{t}\right)^{*}(\sigma)=\sigma_{t}-\sigma_{t_{0}} .
$$

In particular, $\tau_{t_{1}}=\sigma_{t_{1}}-\sigma_{t_{0}}=\alpha$ and $\tau_{t_{0}}=0$. Hence (b) is satisfied.

The proof is complete since it is obvious that (b) implies (a).

Proof of Proposition 1.2. In order to prove that $\operatorname{Alg}^{k}(X)$ is a subgroup of $H_{\mathrm{alg}}^{k}(X, \mathbb{Z} / 2)$ it suffices to show that given $\alpha$ and $\beta$ in $\operatorname{Alg}^{k}(X)$, the sum $\alpha+\beta$ is in $\operatorname{Alg}^{k}(X)$. By Lemma 2.1, there exist compact nonsingular irreducible real algebraic varieties $T$ and $U$, and cohomology classes $\sigma$ in $H_{\mathrm{alg}}^{k}(X \times T, \mathbb{Z} / 2)$ and $\tau$ in $H_{\mathrm{alg}}^{k}(X \times U, \mathbb{Z} / 2)$ such that $\sigma_{t_{0}}=0, \sigma_{t_{1}}=\alpha$ for some $t_{0}, t_{1}$ in $T$ and $\tau_{u_{0}}=0, \tau_{u_{1}}=\beta$ for some $u_{0}, u_{1}$ in $U$. Given $t$ in $T$ and $u$ in $U$, let $i_{t}: X \rightarrow X \times T, j_{u}: X \rightarrow X \times U, e_{(t, u)}: X \rightarrow X \times T \times U$ be the maps defined by $i_{t}(x)=(x, t), j_{u}(x)=(x, u), e_{(t, u)}(x)=(x, t, u)$ for all $x$ in $X$. Denoting by $\pi: X \times T \times U \rightarrow X \times T$ and $\rho: X \times T \times U \rightarrow X \times U$ the canonical projections, we have $\pi \circ e_{(t, u)}=i_{t}$ and $\rho \circ e_{(t, u)}=j_{u}$. Thus, setting $\xi=\pi^{*}(\sigma)+\rho^{*}(\tau)$, we get

$$
\begin{aligned}
\xi_{(t, u)} & =e_{(t, u)}^{*}\left(\pi^{*}(\sigma)+\rho^{*}(\tau)\right) \\
& =\left(\pi \circ e_{(t, u)}\right)(\sigma)+\left(\rho \circ e_{(t, u)}\right)^{*}(\tau) \\
& =i_{t}^{*}(\sigma)+j_{u}^{*}(\tau) \\
& =\sigma_{t}+\tau_{u} .
\end{aligned}
$$

In particular, $\xi_{\left(t_{0}, u_{0}\right)}=\sigma_{t_{0}}+\tau_{u_{0}}=0$ and $\xi_{\left(t_{1}, u_{1}\right)}=\sigma_{t_{1}}+\tau_{u_{1}}=\alpha+\beta$. Hence $\alpha+\beta$ is in $\operatorname{Alg}^{k}(X)$. We proved that $\operatorname{Alg}^{k}(X)$ is a subgroup of $H_{\mathrm{alg}}^{k}(X, \mathbb{Z} / 2)$.

Let $p: X \times T \rightarrow X$ be the canonical projection and set $\eta=\sigma \cup p^{*}(\gamma)$. Since $p \circ i_{t}$ is the identity map of $X$, we get

$$
\eta_{t}=i_{t}^{*}\left(\sigma \cup p^{*}(\gamma)\right)=i_{t}^{*}(\sigma) \cup i_{t}^{*}\left(p^{*}(\gamma)\right)=\sigma_{t} \cup\left(p \circ i_{t}\right)^{*}(\gamma)=\sigma_{t} \cup \gamma .
$$

In particular, $\eta_{t_{0}}=\sigma_{t_{0}} \cup \gamma=0 \cup \gamma=0$ and $\eta_{t_{1}}=\sigma_{t_{1}} \cup \gamma=\alpha \cup \gamma$. Thus $\alpha \cup \gamma$ is in $\operatorname{Alg}^{k+\ell}(X)$.

It remains to prove that $\gamma \times \delta$ is in $\operatorname{Alg}^{\ell+m}(X \times Y)$. By Lemma 2.1, there exist a compact nonsingular irreducible real algebraic variety $T$, two points $t_{0}$ and $t_{1}$ in $T$, and a cohomology class $\theta$ in $H_{\mathrm{alg}}^{m}(Y \times T, \mathbb{Z} / 2)$ such that $\theta_{t_{0}}=0$ and $\theta_{t_{1}}=\delta$. Since 
$\gamma \times \theta=q^{*}(\gamma) \cup r^{*}(\theta)$, where $q: X \times Y \times T \rightarrow X$ and $r: X \times Y \times T \rightarrow Y \times T$ are the canonical projections, it follows that $\gamma \times \theta$ belong to $H_{\mathrm{alg}}^{\ell+m}(X \times Y \times T, \mathbb{Z} / 2)$. For each $t$ in $T$, we have $(\gamma \times \theta)_{t}=\gamma \times \theta_{t}$. In particular, $(\gamma \times \theta)_{t_{0}}=\gamma \times \theta_{t_{0}}=\gamma \times 0=0$ and $(\gamma \times \theta)_{t_{1}}=\gamma \times \theta_{t_{1}}=\gamma \times \delta$. Hence $\gamma \times \delta$ is in $\operatorname{Alg}^{\ell+m}(X \times Y)$.

Proof of Proposition 1.3. (i) Let $\beta$ be an element of $\operatorname{Alg}^{k}(Y)$. By Lemma 2.1, there exist a compact nonsingular irreducible real algebraic variety $T$, two points $t_{0}$ and $t_{1}$ in $T$, and a cohomology class $\tau$ in $H_{\mathrm{alg}}^{k}(Y, \mathbb{Z} / 2)$ such that $\tau_{t_{0}}=0$ and $\tau_{t_{1}}=\beta$. For $t$ in $T$, let $i_{t}: X \rightarrow X \times T$ and $j_{t}: Y \rightarrow Y \times T$ be the maps defined by $i_{t}(x)=(x, t)$ for all $x$ in $X$ and $j_{t}(y)=(y, t)$ for all $y$ in $Y$. Denoting by $i: X \rightarrow X$ the identity map, we have $(f \times i) \circ i_{t}=j_{t} \circ f$. Thus, setting $\sigma=(f \times i)^{*}(\tau)$, we obtain

$$
\sigma_{t}=i_{t}^{*}\left((f \times i)^{*}(\tau)\right)=\left((f \times i) \circ i_{t}\right)^{*}(\tau)=\left(j_{t} \circ f\right)^{*}(\tau)=f^{*}\left(j_{t}(\tau)\right)=f^{*}\left(\tau_{t}\right) .
$$

In particular, $\sigma_{t_{0}}=f^{*}\left(\tau_{t_{0}}\right)=f^{*}(0)=0$ and $\sigma_{t_{1}}=f^{*}\left(\tau_{t_{1}}\right)=f^{*}(\beta)$, and hence $f^{*}(\beta)$ is in $\mathrm{Alg}^{k}(X)$. This completes the proof of (i).

(ii) Let $\alpha$ be an element of $\operatorname{Alg}^{n-k}(X)$. By Lemma 2.1, there exist a compact nonsingular irreducible real algebraic variety $T$, two points $t_{0}$ and $t_{1}$ in $T$, and a cohomology class $\sigma$ in $H_{\mathrm{alg}}^{n-k}(X \times T, \mathbb{Z} / 2)$ such that $\sigma_{t_{0}}=0$ and $\sigma_{t_{1}}=\alpha$.

Given a point $t$ in $T$, let $e_{t}:\{t\} \hookrightarrow T$ be the inclusion map. For any cohomology class $\eta$ in $H^{s}(T, \mathbb{Z} / 2)$, we define the element $\epsilon_{t}(\eta)$ of $\mathbb{Z} / 2$ by setting $\epsilon_{t}(\eta)=1$ if $s=0$ and $e_{t}^{*}(\eta) \neq 0$, and $\epsilon_{t}(\eta)=0$ in all other cases.

For any $\lambda$ in $H^{r}(X, \mathbb{Z} / 2)$ and any $\mu$ in $H^{r}(Y, \mathbb{Z} / 2)$, we have

$$
i_{t}^{*}(\lambda \times \eta)=\epsilon_{t}(\eta) \lambda, \quad j_{t}^{*}(\mu \times \eta)=\epsilon_{t}(\eta) \mu,
$$

where the $i_{t}$ and $j_{t}$ are the maps defined as in (i). If $e$ is the identity map of $T$, then

$$
\begin{aligned}
\left(D_{Y} \circ j_{t}^{*} \circ D_{Y \times T}^{-1} \circ(f \times e)_{*} \circ D_{X \times T}\right)(\lambda \times \eta) & =\left(D_{Y} \circ j_{t}^{*} \circ D_{Y \times T}^{-1} \circ(f \times e)_{*}\right)\left(D_{X}(\lambda) \times D_{T}(\eta)\right) \\
& =\left(D_{Y} \circ j_{t}^{*} \circ D_{Y \times T}^{-1}\right)\left(f_{*}\left(D_{X}(\lambda)\right) \times D_{T}(\eta)\right) \\
& =D_{Y}\left(j_{t}^{*}\left(D_{Y}^{-1}\left(f_{*}\left(D_{X}(\lambda)\right)\right) \times \eta\right)\right) \\
& =D_{Y}\left(\epsilon_{t}(\eta) D_{Y}^{-1}\left(f_{*}\left(D_{X}(\lambda)\right)\right)\right) \\
& =\epsilon_{t}(\eta) f_{*}\left(D_{X}(\lambda)\right) \\
& =f_{*}\left(D_{X}\left(\epsilon_{t}(\lambda) \lambda\right)\right) \\
& =\left(f_{*} \circ D_{X} \circ i_{t}^{*}\right)(\lambda \times \eta) .
\end{aligned}
$$

Since $r$ and $s$ are arbitrary, it follows from Künneth's theorem for cohomology that

$$
D_{Y} \circ j_{t}^{*} \circ D_{Y \times T}^{-1} \circ(f \times e)_{*} \circ D_{X \times T}=f_{*} \circ D_{X} \circ i_{t}^{*}
$$

as homomorphisms from $H^{*}(X \times T, \mathbb{Z} / 2)$ into $H_{*}(Y, \mathbb{Z} / 2)$, and hence

$$
j_{t}^{*} \circ D_{Y \times T}^{-1} \circ(f \times e)_{*} \circ D_{X \times T}=D_{Y}^{-1} \circ f_{*} \circ D_{X} \circ i_{t}^{*} .
$$

Setting now $\tau=\left(D_{Y \times T}^{-1} \circ(f \times e)_{*} \circ D_{X \times T}\right)(\sigma)$, we obtain

$$
\tau_{t}=j_{t}^{*}(\tau)=\left(D_{Y}^{-1} \circ f_{*} \circ D_{X} \circ i_{t}^{*}\right)(\sigma)=\left(D_{Y}^{-1} \circ f_{*} \circ D_{X}\right)\left(\sigma_{t}\right)
$$


In particular,

$$
\begin{aligned}
& \tau_{t_{0}}=\left(D_{Y}^{-1} \circ f_{*} \circ D_{X}\right)\left(\sigma_{t_{0}}\right)=\left(D_{Y}^{-1} \circ f_{*} \circ D_{X}\right)(0)=0 \\
& \tau_{t_{1}}=\left(D_{Y}^{-1} \circ f_{*} \circ D_{X}\right)\left(\sigma_{t_{1}}\right)=\left(D_{Y}^{-1} \circ f_{*} \circ D_{X}\right)(\alpha) .
\end{aligned}
$$

Hence $\left(D_{Y}^{-1} \circ f_{*} \circ D_{X}\right)(\alpha)$ is in $\operatorname{Alg}^{p-k}(Y)$, and the proof of (ii) is complete.

3. Proofs of the theorems. We begin with the following result.

LEMMA 3.1. Let $X$ be a compact nonsingular real algebraic variety of dimension $n$. Then for any cohomology class $\alpha$ in $\operatorname{Alg}^{n}(X)$, one has $\langle\alpha,[X]\rangle=0$.

Proof. Choose a finite subset $S$ of $X$ representing the homology class $D_{X}(\alpha)=\alpha \cap[X]$ in $H_{0}(X, \mathbb{Z} / 2)$. By [7, p. 239], $\langle\alpha,[X]\rangle=\epsilon(\alpha \cap[X])$, where $\epsilon: H_{0}(X, \mathbb{Z} / 2) \rightarrow \mathbb{Z} / 2$ is the augmentation homomorphism. Hence, denoting by $\# S$ the number of elements of $S$, we get

$$
\langle\alpha,[X]\rangle=\# S(\bmod 2) .
$$

In order to complete the proof it suffices to show that \#S is an even integer.

Suppose that $\# S$ is an odd integer. We obtain a contradiction as follows. Let $Y$ be a real algebraic variety consisting of one point and let $f: X \rightarrow Y$ be the unique possible map. Obviously, $\left(D_{Y}^{-1} \circ f_{*} \circ D_{X}\right)(\alpha) \neq 0$ in $H^{0}(Y, \mathbb{Z} / 2) \cong \mathbb{Z} / 2$. On the other hand, by Proposition 1.3(ii), $\left(D_{Y}^{-1} \circ f_{*} \circ D_{X}\right)(\alpha)$ is in $\operatorname{Alg}^{0}(Y)$. However, since $Y$ consists of one point, it follows from the definition that $\operatorname{Alg}^{0}(Y)=0$. Thus we have a contradiction and the proof is complete.

Proof of Theorem 1.4. By assumption, $\alpha_{1}-\alpha_{2}$ is in $\operatorname{Alg}^{k}(X)$ and $\beta_{1}-\beta_{2}$ is in $\operatorname{Alg}^{\ell}(X)$. Therefore, in view of Proposition 1.2, $\left(\alpha_{1}-\alpha_{2}\right) \cup \beta_{1}$ and $\alpha_{2} \cup\left(\beta_{1} \cup \beta_{2}\right)$ are in $\operatorname{Alg}^{k+\ell}(X)$. Hence

$$
\begin{aligned}
& \left\langle\alpha_{1} \cup \beta_{1},[X]\right\rangle-\left\langle\alpha_{2} \cup \beta_{1},[X]\right\rangle=\left\langle\left(\alpha_{1}-\alpha_{2}\right) \cup \beta_{1},[X]\right\rangle=0, \\
& \left\langle\alpha_{2} \cup \beta_{1},[X]\right\rangle-\left\langle\alpha_{2} \cup \beta_{2},[X]\right\rangle=\left\langle\alpha_{2} \cup\left(\beta_{1}-\beta_{2}\right),[X]\right\rangle=0,
\end{aligned}
$$

where the last equality in either line is a consequence of Lemma 3.1. It follows that $\left\langle\alpha_{1} \cup \beta_{1},[X]\right\rangle=\left\langle\alpha_{2} \cup \beta_{2},[X]\right\rangle$, which is equivalent to $\alpha_{1} \bullet \beta_{1}=\alpha_{2} \bullet \beta_{2}$. The proof is complete.

The proof of Theorem 1.7 requires some preparation. All manifolds we use will be smooth (of class $\mathcal{C}^{\infty}$ ), paracompact and without boundary. Let $M$ be a smooth manifold and let $N$ be a smooth submanifold of $M$. Assume that $N$ is a closed subset of $M$. We denote by $\tau_{N}^{M}$ the Thom class of $N$ in $M$; thus $\tau_{N}^{M}$ is in $H^{k}(M, M \backslash N ; \mathbb{Z} / 2)$, where $k=\operatorname{dim} M-\operatorname{dim} N$. If $N=\{x\}$, we shall write $\tau_{x}^{M}$ instead of $\tau_{\{x\}}^{M}$. Clearly, $\tau_{x}^{M}$ is just the unique generator of the group $H^{m}(M, M \backslash\{x\}, \mathbb{Z} / 2) \cong \mathbb{Z} / 2, m=\operatorname{dim} M$. As usual, $w_{i}(M)$ will denote the $i$ th Stiefel-Whitney class of $M$.

Given a topological space $T$, we let $\epsilon_{T}: H_{0}(T, \mathbb{Z} / 2) \rightarrow \mathbb{Z} / 2$ denote the augmentation homomorphism.

Proof of Theorem 1.7. Let $n=\operatorname{dim} X, p=\operatorname{dim} Y$, and $k=n-p$. For any point $y$ in $Y$, let $\beta_{y}$ denote the cohomology class in $H^{p}(Y, \mathbb{Z} / 2)$ Poincare dual to the homology class in $H_{0}(Y, \mathbb{Z} / 2)$ represented by $y$. By Example 1.1, given $y_{1}$ and $y_{2}$ 
in $Y$, the cohomology class $\beta_{y_{1}}-\beta_{y_{2}}$ belongs to $\operatorname{Alg}^{p}(Y)$. In view of Proposition 1.3(i), $f^{*}\left(\beta_{y_{1}}-\beta_{y_{2}}\right)=f^{*}\left(\beta_{y_{1}}\right)-f^{*}\left(\beta_{y_{2}}\right)$ is in $\operatorname{Alg}^{p}(X)$ and hence Theorem 1.4 implies that

$$
\alpha \bullet f^{*}\left(\beta_{y_{1}}\right)=\alpha \bullet f^{*}\left(\beta_{y_{2}}\right)
$$

for every cohomology class $\alpha$ in $H_{\mathrm{alg}}^{k}(X, \mathbb{Z} / 2)$. It is known that $w_{i}(X)$ is in $H_{\mathrm{alg}}^{i}(X, \mathbb{Z} / 2)$ for all $i \geq 0[2,3]$. Thus, given nonnegative integers $i_{1}, \ldots, i_{r}$ with $i_{1}+\cdots+i_{r}=k$, we have

$$
\left(w_{i_{1}}(X) \cup \ldots \cup w_{i_{r}}(X)\right) \bullet f^{*}\left(\beta_{y_{1}}\right)=\left(w_{i_{1}}(X) \cup \ldots \cup w_{i_{r}}(X)\right) \bullet f^{*}\left(\beta_{y_{2}}\right)
$$

Let us set

$$
n_{i_{1} \ldots i_{r}}(f, y)=\left(w_{i_{1}}(X) \cup \ldots \cup w_{i_{r}}(X)\right) \bullet f^{*}\left(\beta_{y}\right)
$$

Note that

$$
n_{i_{1} \ldots i_{r}}(f, y)=0 \text { for } y \text { in } Y \backslash f(X)
$$

since $y$ in $Y \backslash f(X)$ implies $f^{*}\left(\beta_{y}\right)=0$.

If $y$ in $f(X)$ is a regular value of $f$, then $f^{-1}(y)$ is a smooth submanifold of $X$ of dimension $k$. We assert

$$
n_{i_{1} \ldots i_{r}}(f, y)=\left\langle w_{i_{1}}\left(f^{-1}(y)\right) \cup \ldots \cup w_{i_{r}}\left(f^{-1}(y)\right),\left[f^{-1}(y)\right]\right\rangle .
$$

Suppose that (3) holds. If $y_{1}$ and $y_{2}$ are regular values of $f$, then (1), (2), and (3) guarantee that $f^{-1}\left(y_{1}\right)$ and $f^{-1}\left(y_{2}\right)$ have the same Stiefel-Whitney numbers. Hence, by Thom's theorem [11], the smooth manifolds $f^{-1}\left(y_{1}\right)$ and $f^{-1}\left(y_{2}\right)$ are cobordant. Thus it remains to prove (3).

In order to simplify notation set $F=f^{-1}(y)$. Let $\bar{f}:(X, X \backslash F) \rightarrow(Y, Y \backslash\{y\})$ be the map defined by $f$. Since $y$ is a regular value of $f$, we have

$$
\bar{f}^{*}\left(\tau_{y}^{Y}\right)=\tau_{F}^{X}
$$

Moreover the following diagram is commutative:

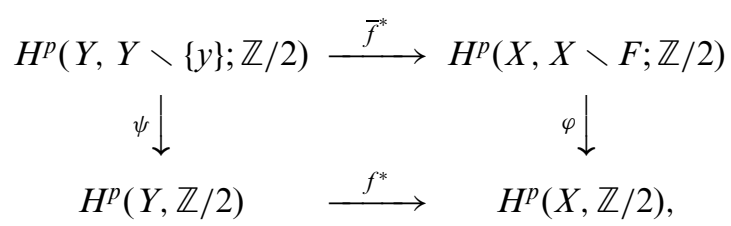

where $\varphi$ and $\psi$ are the canonical homomorphisms. Since $\psi\left(\tau_{y}^{Y}\right)=\beta_{y}$, it follows that

$$
f^{*}\left(\beta_{y}\right)=f^{*}\left(\psi\left(\tau_{y}^{Y}\right)\right)=\varphi\left(\bar{f}^{*}\left(\tau_{y}^{Y}\right)\right)=\varphi\left(\tau_{F}^{X}\right) .
$$

Note that if $e: F \hookrightarrow X$ is the inclusion map, then

$$
\left\langle\alpha \cup \varphi\left(\tau_{F}^{X}\right),[X]\right\rangle=\left\langle e^{*}(\alpha),[F]\right\rangle
$$


for every cohomology class $\alpha$ in $H^{p}(X, \mathbb{Z} / 2)$. Indeed, (5) can be proved by direct computation:

$$
\begin{aligned}
\left\langle\alpha \cup \varphi\left(\tau_{F}^{X}\right),[X]\right\rangle & =\epsilon_{X}\left(\left(\alpha \cup \varphi\left(\tau_{F}^{X}\right)\right) \cap[X]\right) \\
& =\epsilon_{X}\left(\alpha \cap\left(\varphi\left(\tau_{F}^{X}\right) \cap[X]\right)\right) \\
& =\epsilon_{X}\left(\alpha \cap e_{*}([F])\right) \\
& =\epsilon_{X}\left(e_{*}\left(e^{*}(\alpha) \cap[F]\right)\right) \\
& =\epsilon_{F}\left(e^{*}(\alpha) \cap[F]\right) \\
& =\left\langle e^{*}(\alpha),[F]\right\rangle,
\end{aligned}
$$

where the third equality holds since $\varphi\left(\tau_{F}^{X}\right) \cap[X]=e_{*}([F])[10$, Problem 11.C], the fifth equality is a consequence of naturality of augmentation, and the other equalities are standard properties of the $\cup, \cap$, and $\langle$,$\rangle products [7].$

Furthermore, since the normal vector bundle of $F$ in $X$ is trivial, we have $e^{*}\left(w_{i}(X)\right)=w_{i}(F)$ for all $i \geq 0$, and hence

$$
e^{*}\left(w_{i_{1}}(X) \cup \ldots \cup w_{i_{r}}(X)\right)=w_{i_{1}}(F) \cup \ldots \cup w_{i_{r}}(F) .
$$

Now, making use of (4), (5), and (6), we get

$$
\begin{aligned}
n_{i_{1} \ldots i_{r}}(f, y) & =\left\langle w_{i_{1}}(X) \cup \ldots \cup w_{i_{r}}(X) \cup f^{*}\left(\beta_{y}\right),[X]\right\rangle \\
& =\left\langle w_{i_{1}}(X) \cup \ldots \cup w_{i_{r}}(X) \cup \varphi\left(\tau_{F}^{X}\right),[X]\right\rangle \\
& =\left\langle e^{*}\left(w_{i_{1}}(X) \cup \ldots \cup w_{i_{r}}(X)\right),[F]\right\rangle \\
& =\left\langle w_{i_{1}}(F) \cup \ldots \cup w_{i_{r}}(F),[F]\right\rangle,
\end{aligned}
$$

which proves (3). Hence the proof is complete.

\section{REFERENCES}

1. M. Abánades and W. Kucharz, Algebraic equivalence of real algebraic cycles, Ann. Inst. Fourier (Grenoble) 49 (1999), 1797-1804.

2. S. Akbulut and H. King, Submanifolds and homology of nonsingular algebraic varieties, Amer. J. Math. 107 (1985), no. 1, 45-83.

3. R. Benedetti and A. Tognoli, Remarks and counterexamples in the theory of real vector bundles and cycles, in Géométrie algébrique réelle et formes quadratiques, Lecture Notes in Math. No. 959 (Springer-Verlag, 1982), 198-211.

4. J. Bochnak, M. Coste and M.-F. Roy, Real algebraic geometry, Ergebnisse der Math. und ihrer Grenzgeb. Folge (3), Vol. 36, (Springer-Verlag, 1998).

5. J. Bochnak and W. Kucharz, On approximation of smooth submanifolds by nonsingular real algebraic subvarieties, Ann. Sci. École Norm. Sup. (4), to appear.

6. A. Borel and A. Haefliger, La classe d'homologie fondamentale d'un espace analytique, Bull. Soc. Math. France 89 (1961), 461-513.

7. A. Dold, Lectures on algebraic topology, Grundlehren Math. Wiss. Vol. 200 (SpringerVerlag, 1972).

8. W. Kucharz, Algebraic equivalence and homology classes of real algebraic cycles, Math. Nachr. 180 (1996), 135-140.

9. W. Kucharz, Algebraic cycles and algebraic models of smooth manifolds, J. Algebraic Geometry 11 (2002), 101-127.

10. J. Milnor and J. Stasheff, Characteristic classes, Ann. of Math. Studies 76 (Princeton Univ. Press, Princeton, New Jersey, 1974). 1958)

11. R. Stong, Notes on cobordism theory, Princeton Math. Notes (Princeton Univ. Press, 